\title{
OBSERVAÇÕES SOBRE O COMPORTAMENTO DE LIOLAEMUS OCCIPITALIS EM CATIVEIRO (SAURIA, TROPIDURIDAE)
}

\author{
Clóvis Souza Bujes ${ }^{1}$ \\ Laura Verrastro ${ }^{1}$
}

\begin{abstract}
OBSERVATIONS ON THE BEHAVIOR OF LIOLAEMUS OCCIPITALIS IN CAPTIVITY (SAURIA, TropiduridaE). Liolaemus occipitalis (Boulenger, 1885) is an abundant lizard found along the coastal dunes of Rio Grande do Sul, Brazil. Fourteen individuals were manually captured in the dunes of Quintão beach (Palmares do Sul, Rio Grande do Sul) and studied in captivity from 23/VII/92 to 30/VII/93. They were kept in a terrarium with sand substrate and vegetation collected in their habitat. Snout-vent length (SVL) and weighted were measured weekly. They received Tenebrio molitor (Linnaeus, 1758) worms as food; water was abundant. Combats between males for food and territory, and other behavioral displays were observed, like copulation and nesting behaviors. Growth curves for each lizard were analyzed.

KEY WORDS. Tropiduridae, Liolaemus, agonistic behavior, captivity, growing rate, reproductive behavior
\end{abstract}

Liolaemus occipitalis Boulenger, 1885 é um lagarto abundante nas dunas costeiras do Rio Grande do Sul e restrito a este tipo de ambiente, apresentando condições peculiares de adaptação. O comprimento rostro-anal médio dos machos é de $60,2 \mathrm{~mm}$ e das fêmeas, $53,2 \mathrm{~mm}$. Possui padrão de coloração críptico, costumando enterrar-se em tocas ou na areia superficial. A reprodução acontece entre setembro e março, e sua dieta preferencial é insetívora(VERRASTRO \& KRAUSE 1994).

Desde 1986 vem-se desenvolvendo estudos de caráter biológico e ecológico nas populações do litoral norte do Estado (VERRASTRO \& KRAUSE 1994), enfocando a reprodução, dieta, crescimento, ritmo de atividade e alguns parâmetros populacionais.

Outros trabalhos realizados com esta espécie foram de caráter taxonômico, biogeográfico e osteológico (VANZOLINI \& AB’SABER 1968; MÜLLER \& STEINGER 1977; Keller \& KRAUSE 1988; SiMÕES-LOPES \& KRAUSE 1986).

O presente estudo foi uma tentativa de criar este lagarto em cativeiro, visando conseguir informações referentes à cópula, período de retenção de ovos, oviposição, período de incubação e construção de tocas, devido às dificuldades de obtenção destes dados na natureza.

1) Departamento de Zoologia, Instituto de Biociências, Universidade Federal do Rio Grande do Sul. 90046-900 Porto Alegre, Rio Grande do Sul, Brasil.

E-mail: csbujes@conex.com.br ou lauraver@vortex.ufrgs.br 


\section{MATERIAL E MÉTODOS}

Durante o período de 23/VII/92 e 30/VII/93 foram mantidos em laboratório 14 exemplares de L. occipitalis (seis machos adultos, dois machos jovens, quatro fêmeas adultas, duas fêmeas jovens) que foram capturados manualmente, em diferentes épocas do ano (Tab. I), nas dunas da praia do Quintão ( $30^{\circ} 24^{\prime} \mathrm{S}, 50^{\circ} 17^{\prime} \mathrm{W}$ Gr), Palmares do Sul, Rio Grande do Sul.

Tabela I. Distribuição dos exemplares de $L$. occipitalis pelos terrários e suas respectivas datas de captura e morte. (MA) Macho adulto, (MAD) macho adulto dominante, (MJ) macho jovem, (FA) fêmea adulta, (FJ) fêmea jovem.

\begin{tabular}{|c|c|c|c|c|}
\hline Exemplar & Sexo & Terrário & Data de captura & Data de morte \\
\hline Lo01 & MA & 1 & 23-VII-92 & 24-IV-93 \\
\hline LoO2 & MA & 1 & 23-VII-92 & $27-X-92$ \\
\hline Lo03 & FJ & 1 & 05-VIII-92 & $15-11-92$ \\
\hline Lo04 & FJ & 1 & 05-VIII-92 & $29-X-92$ \\
\hline Lo05 & MJ & 1 & 05-VIII-92 & $07-X 11-92$ \\
\hline Lo06 & MA & 2 & 05-VIII-92 & $25-111-93$ \\
\hline Lo07 & MAD & 2 & 05-VIII-92 & 04-VIII-93 \\
\hline Lo08 & MAD & 1 & 05-VIII-92 & 04-VIII-93 \\
\hline Lo09 & MAD & 3 & 05-VIII-92 & 04-VIII-93 \\
\hline Lo10 & MJ & 3 & 05-VIII-92 & 04-VIII-93 \\
\hline Lo11 & FA & 3 & $07-1 X-92$ & $17-\mathrm{XI}-92$ \\
\hline Lo12 & FA & 3 & $07-1 X-92$ & $28-$ VI-93 \\
\hline Lo13 & FA & 2 & $07-1 X-92$ & 04-VIII-93 \\
\hline Lo14 & FA & 2 & $12-X \mid 1-92$ & 04-VIII-93 \\
\hline
\end{tabular}

Cada lagarto foi marcado individualmente através do método de amputação de falanges (VERRASTRO \& KRAUSE 1994). Semanalmente, eram pesados com balança manual (precisão de $0,1 \mathrm{~g}$ ), sendo realizadas medidas do comprimento rostro-anal (CRA) com paquímetro (precisão $0,1 \mathrm{~mm}$ ).

Os animais foram mantidos em três terrários de vidro com as seguintes dimensões: 1) $75 \times 50 \times 55 \mathrm{~cm}$; 2) $50 \times 50 \times 45 \mathrm{~cm}$; 3) $50 \times 30 \times 30 \mathrm{~cm}$. Os terrários recebiam luz natural através de uma janela, bem como luz fornecida por lâmpadas incandescentes de $40 \mathrm{~W}$, acopladas às tampas de madeira e tela que cobriam os mesmos. Estas lâmpadas permaneciam acesas por, aproximadamente oito horas no inverno e seis horas no verão. Como substrato, utilizou-se areia (um terço da altura do terrário) coletada no próprio local de captura. Alguns vegetais da área foram acondicionados nos terrários. A água para os animais era fornecida em abundância, acrescida de algumas gotas de azul de metileno e substituída a cada dois dias. O alimento [larvas de Tenebrio molitor (Linnaeus, 1758)] era fornecido a cada dois dias, na proporção de três larvas por lagarto. Durante os meses de novembro e dezembro, as larvas passaram para o estágio adulto. Os adultos não eram consumidos pelos lagartos, assim a proporção nesse período caiu para uma larva por lagarto. Havia registro diário da temperatura nos terrários, através da leitura de um termômetro de mercúrio (precisão de $1^{\circ} \mathrm{C}$ ) posicionado $5 \mathrm{~cm}$ acima da superfície da areia. Obteve-se uma média de $25^{\circ} \mathrm{C}\left(11^{\circ} \mathrm{C}-37^{\circ} \mathrm{C}\right)$. Não houve controle da umidade nos terrários. 


\section{RESULTADOS E DISCUSSÃO}

Devido a sua natureza territorialista e estando habituado a amplas áreas de domicílio, cerca de $732 \mathrm{~m}^{2}$ para machos e $307 \mathrm{~m}^{2}$ para fêmeas (VERRASTRO \& KRAUSE 1994), L. occipitalis demonstrou comportamento típico de estresse em cativeiro. O primeiro obstáculo enfrentado foi a aceitação do alimento; o segundo, a disputa por espaço e, o terceiro as disputas pelo alimento escasso.

Foram necessárias duas semanas para que os lagartos se alimentassem pela primeira vez. Apenas larvas de T. molitor, muito similares às larvas encontradas por eles em seu habitat, foram aceitas como alimento. Em uma ocasião foram oferecidas larvas de Diptera, tendo sido rejeitadas pelos lagartos. Pupas e adultos de T. molitor também não foram aceitos, os adultos possivelmente, devido aos élitros que possuem.

Observou-se, desde o início, hierarquia entre os indivíduos de cada terrário. Os adultos impediam os jovens de ocupar espaços ao sol ou sob o calor da lâmpada e os mantinham distantes do alimento. Da mesma forma, alguns machos subjugavam a outros de similar tamanho, bem como as fêmeas, através dessas disputas por espaço e alimento.

Registrou-se comportamento de sinalização, comum à família, seja para marcação de território, disputa de alimento ou para cortejo a parceiros (VITT \& LACHER 1981; TOLLESTRUP 1981). Nas sinalizações, o lagarto flexionava de três a quatro vezes as patas anteriores que, em seguida, eram mantidas eretas; elevando o tronco levemente, sacudia simultaneamente a cabeça para cima e para baixo, com movimentos rápidos do pescoço. Este display era repetido por outros lagartos que estavam próximos. A seguir, ocorriam disputas que variavam desde uma simples perseguição, até lutas propriamente ditas. Tais lutas consistiam de mordidas na região gular, nos fêmures e nos flancos. Em muitas delas, os lagartos chegavam a ferir-se mortalmente, como o indivíduo Lo01 encontrado morto com graves lesões, inclusive fraturas. Os indivíduos que não entravam em luta com o lagarto dominante se enterravam superficialmente na areia ou se escondiam entre a vegetação. $\mathrm{O}$ lagarto dominante mantinha sempre a preferência pelo alimento e pelas fêmeas.

A dificuldade de adaptação ao cativeiro também refletiu-se nas curvas de crescimento e peso (Figs 1,2). Pode-se observar que em geral os indivíduos mantinham um peso estável nas primeiras semanas. Após as primeiras semanas de cativeiro, começavam a alimentar-se, aumentando seu peso. Aos três meses a queda do peso foi generalizada, possivelmente, devido ao estresse do espaço reduzido. Aqueles indivíduos que melhor se adaptaram (Lo07, Lo08 e Lo09, machos adultos dominantes, ver figura 1) recuperaram seu peso e mantiveram valores compatíveis com o verificado na natureza para seu comprimento. Os indivíduos Lo02, macho adulto, Lo05, macho jovem, e Lo03 e Lo04, fêmeas jovens, ficaram prejudicados na obtenção de alimento, ou deixaram de alimentar-se, chegando à morte, possivelmente por serem dominados. Em relação ao comprimento rostro-anal, os lagartos menores cresceram, no início do experimento, mas a falta de alimento e a competitividade não permitiu que crescessem mais. Os indivíduos que permaneceram vivos até o final do cativeiro (Tab. I), mantiveram suas curvas de crescimento normais, como as verificadas por VERRASTRO \& KRAUSE (1994) na natureza, para indivíduos 

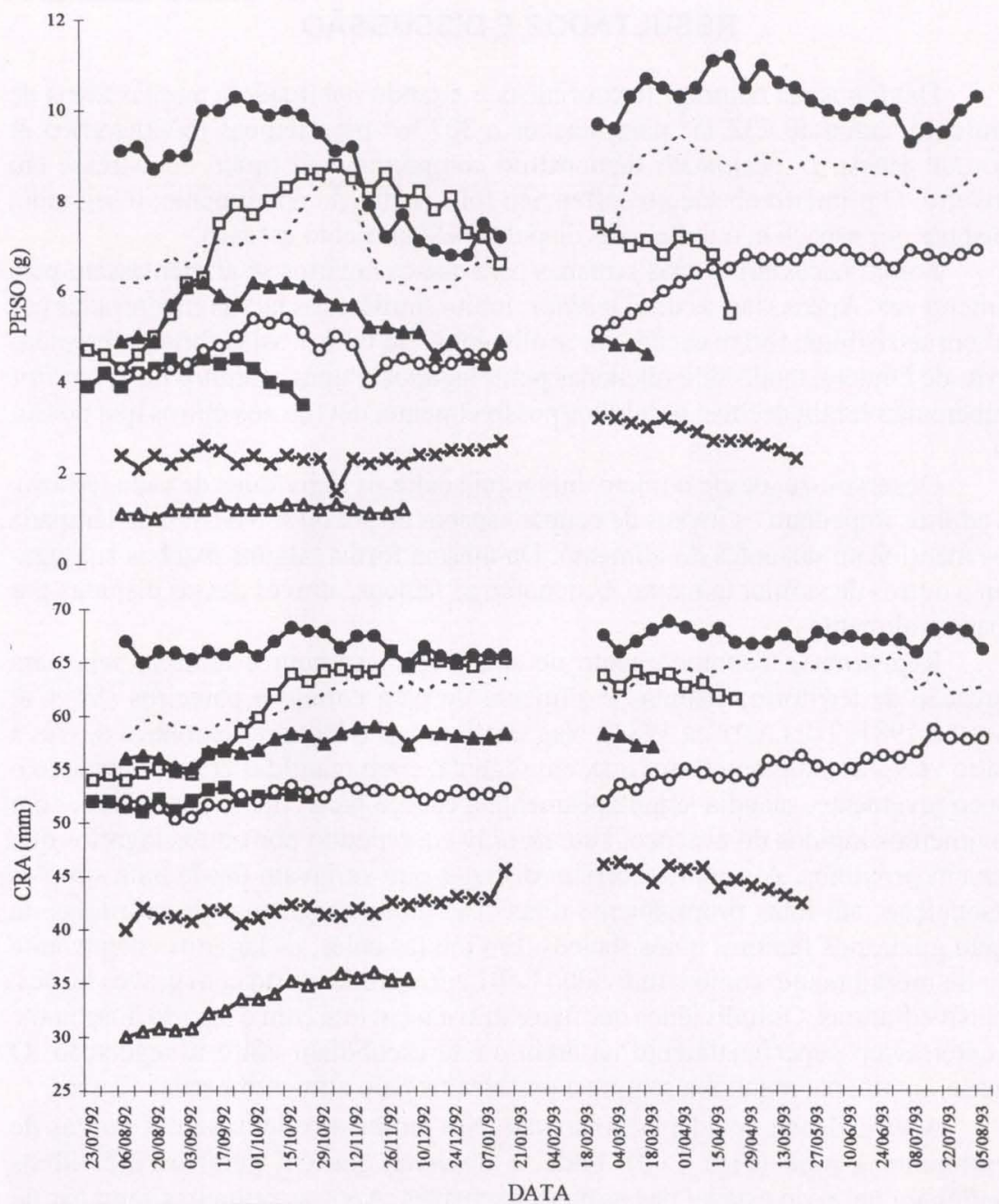

Fig. 1. Peso e comprimento rostro-anal (CRA) de machos $L$. occipitalis mantidos em cativeiro. (Quadrados vazados) Lo01, (quadrados cheios) Lo02, (triângulos vazados) Lo05, (triângulos cheios) Lo06, (círculos vazados) L007, (círculos cheios) Lo08, (linha interrompida) Lo09, (cruzes) Lo10.

adultos. A escassez de alimento ocorrida a partir de novembro/dezembro de 1992 pode ter afetado negativamente o crescimento dos lagartos, levando inclusive à perda de peso.

Na natureza, foi determinada uma estratégia alimentar de "senta-e-espera" (VERRASTRO \& KRAUSE 1994). No cativeiro, foi verificado que estes animais permanecem imóveis e, com um rápido e preciso movimento de cabeça, abocanham a presa no momento que esta se aproxima. 

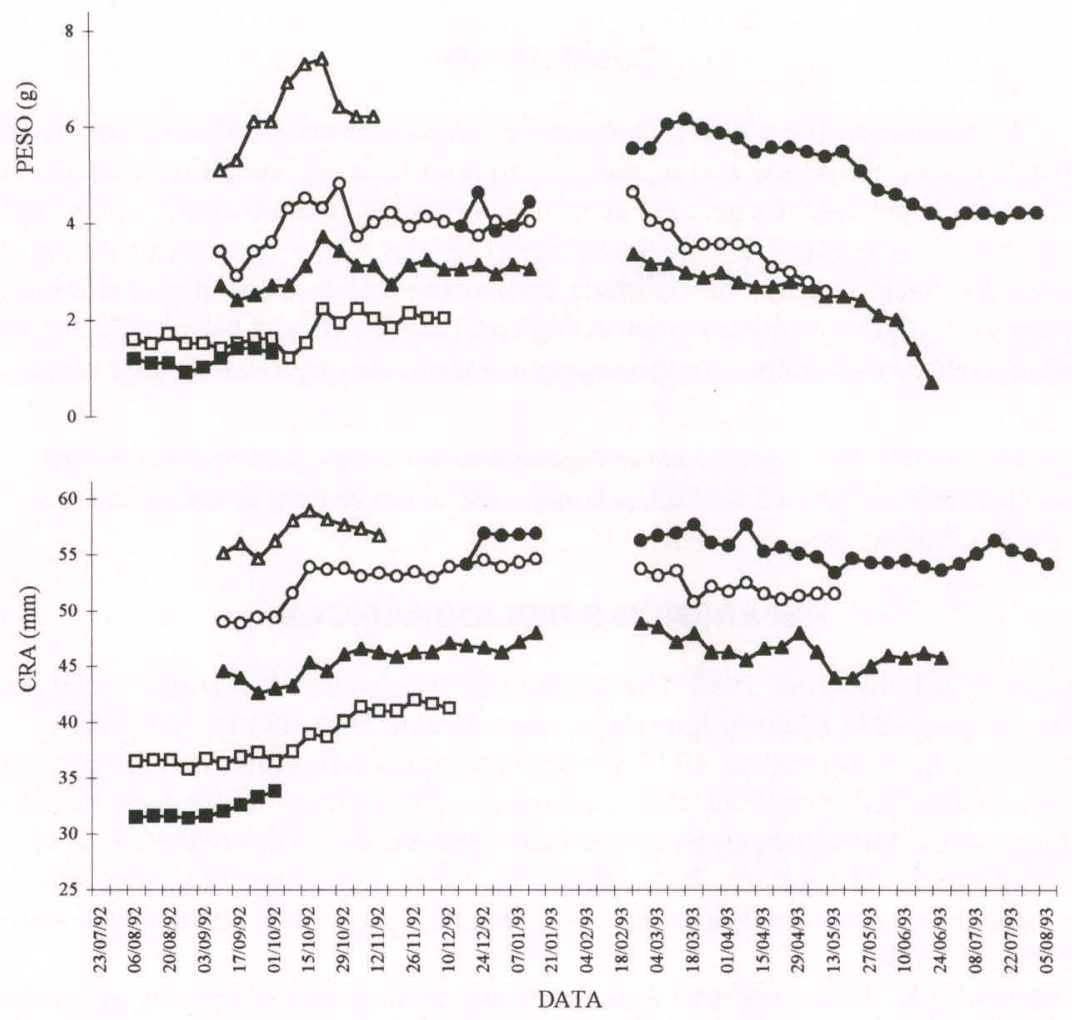

Fig. 2. Peso e comprimento rostro-anal (CRA) de fêmeas $L$. occipitalis mantidas em cativeiro. (quadrados vazados) Lo03, (quadrados cheios) Lo04, (triângulos vazados) Lo11, (triângulos cheios) Lo12, (círculos vazados) Lo13, (círculos cheios) Lo14.

Em 13 de novembro de 1992, obteve-se registro direto de uma cópula entre os indivíduos Lo09 (macho dominante do terrário 3) e Lo11. As fêmeas Lo12 e Lo14 ficaram grávidas no cativeiro, chegando a desovar um ovo cada. As posturas realizadas nos terrários tornaram-se inviáveis, ou porque foram feitas sobre a areia e os ovos rapidamente desidratavam, ou eram destruídos pelo macho dominante (o macho Lo09 foi encontrado com um dos ovos mastigado em sua boca). A fêmea Lo13 que foi trazida grávida para o cativeiro, possivelmente reabsorveu seus ovos, uma vez que não realizou posturas.

Na natureza, L. occipitalis constrói tocas que, entre outras funções, serve de proteção contra predadores e contra as altas temperaturas, além de manter o microclima ideal ao desenvolvimento dos ovos (VERRASTRO \& KRAUSE 1994). As tocas construídas no cativeiro foram utilizadas somente como abrigo e nunca para a postura dos ovos. Estes foram simplesmente depositados sobre a superfície da areia.

Futuramente pretende-se realizar outra tentativa de manutenção da espécie em cativeiro, utilizando-se terrários ao ar livre, para minimizar os problemas relacionados ao ambiente (temperatura, luz, umidade, espaço e outros). 


\section{CONCLUSÃO}

Constatou-se que o lagarto tropidurídeo Liolaemus occipitalis, nas condições proporcionadas, não se adaptou ao cativeiro, apresentando dificuldades na aceitação do alimento e na sobrevivência em espaços confinados. Mesmo assim, apresentou comportamentos comuns à espécie na natureza, tais como construção de tocas, sinalização relacionada à territorialidade, cortejo, oviposição e disputa por alimento. Solucionados alguns problemas metodológicos, esta abordagem permitirá a análise detalhada destes e de outros comportamentos raramente observados na natureza.

AGRADECIMENTOS. Agradecemos ao Departamento de Zoologia da Universidade Federal do Rio Grande do Sul e a FAPERGS, pelo apoio dado através de Bolsa de Recém-Mestre e Auxílio a Pesquisa, processo $N^{\circ} 93.1131 .8$.

\section{REFERÊNCIAS BIBLIOGRÁFICAS}

KELLER, C. \& L. KRAUSE. 1988. The appendicular skeleton of Liolaemus occipitalis Boulenger, 1885 (Sauria, Iguanidae). Rev. Brasil. Biol. 46 (4): 727-740.

MÜLler, P. \& H. STEINGER. 1977. Evolutionsgeschwindiagkeit, Verbreitung und Verwandtschaft brasilianischer Erdleguane der Gattung Liolaemus (Sauria, Iguanidae). Mitteilungen Biogeog. Univ. Saarlandes, Saarbrükent, 9: 1-17.

Simões-Lopes, P.C. DE A. \& L. Krause. 1986. Osteologia do sincrânio de Liolaemus occipitalis Boulenger, 1885 (Sauria, Iguanidae). Revta bras. Zool. 5 (4): 491-508.

Tollestrup, K. 1981. The social behavior and display of two species of horned lizard, Phrynosoma platyrhinos and Phrynosoma coronatum. Herpetologica, Johnson City, 37 (3): 130-141.

VAnZOlini, P.E. \& A.N. AB'SABER. 1968. Divergente rate in South American lizard of the genus Liolaemus (Sauria, Iguanidae). Papéis Avuls Dept. Zool., São Paulo, 21 (21): 205-208.

Verrastro, L. \& L. Krause. 1994. Analysis of growth in a population of Liolaemus occipitalis Boul. 1885, from the coastal sand-dunes of Tramandaí, RS, Brazil (Reptilia-Tropiduridae). Studies Neotrop. Fauna Environ. 29 (2): 99-111.

VITT, L.J. \& T.E. LACHER JR. 1981. Behavior, habitat, diet and reproduction of the iguanid lizard Polychrus acutirostris in the caatinga of northestern Brazil. Herpetologica 37 (1): 53-63.

Recebido em 15.V.1997; aceito em 03.XI.1998. 